\title{
Ecological footprint: a way to assess the impact of tourists' choices at the local scale
}

\author{
V. Castellani \& S. Sala \\ University of Milano Bicocca, \\ Department of Environmental Science, Italy
}

\begin{abstract}
Ecological footprint (EF) is a method developed to assess the sustainability of different consumption patterns and to address human choice in daily life. Considering that different kinds of tourism can have different impacts on the environment (due, for instance, to energy consumption or to the extension of built-up land), there is the need for development of a valuable tool to compare sustainability of different issues related to tourism. In this study, the EF method was fitted to the Italian situation and to an assessment of the potential impact of tourism at the local scale, underlining how tourists' choices could affect the quality of the environment in a tourist destination.

The presented case study consists of the development of a framework, based on the EF method, to assess the ecological costs of different kinds of holiday, related also to the accommodation type chosen by tourists. Through model implementation, it is possible to calculate the EF of one overnight stay for each kind of accommodation. The model was applied to a specific destination in Italy, Alpi Lepontine, to assess the EF of tourism in this area, and to evaluate the sustainability of the destination, comparing the EF of tourists and residents with local biocapacity. The aim of the study is to evaluate the current situation, and to identify solutions that assure the development of tourism with fewer environmental costs (e.g. a network of small hospitality structures, sustainable mobility).

The results of this work represent not only supportive information for decision makers but also a useful tool to raise environmental consciousness among tourists.
\end{abstract}

Keywords: ecological footprint, sustainable tourism, tourist impact, accommodation impact. 


\section{Introduction}

Tourist activities can be a positive element for the local economy of tourist destinations, but can also generate some externalities (positive or, more frequently, negative) that are not included in the economic balance. The impact that is more evident is the one on the natural environment, but there are also some possible effects on the social and economic dynamics of the local community that have to be considered in a sustainable vision. Therefore, it is important that tourism planning made by local decision makers is intended to assure a good level of conservation of natural resources and to mitigate the impacts that tourism necessarily involves. If managed in a responsible and sustainable way, tourism can be a motivating force to the conservation of local heritage; on the other hand, if the strategy adopted for tourism development has only the aim of obtaining huge and immediate economic results through the uncontrolled growth of tourist flow, this will bring a rapid exploitation of the destination, which, after a short period, will become spoilt and no longer be attractive.

Impacts related to tourism activities can be divided into two main categories (May [4]).

a) impacts due to the building of hospitality structures and tourism facilities;

b) impacts due to the presence of tourists and, generally, to the fruition of the area.

a) The first class consists of all impacts due to the building of hotels, restaurants, camping sites and tourist facilities, which can be summarized as: loss of soil previously used for agriculture, pasture or other activities; the necessity to build new roads to connect new tourist structures or to improve and enlarge existing roads to make them adequate for an increased level of traffic. It is important to consider that this kind of impact is persistent, because tourist structures, often scaled for the wider tourist flow of the destination, stay in the territory, even if they are almost empty during the whole year.

b) The presence of tourists can generate two main kinds of problems: the production of solid and liquid wastes (that imply a cost for the disposal which is paid by the local community and needs the organisation of a service of collection dimensioned with reference to the maximum volume generated during the year, i.e. in the tourist season); the possibility of conflict between residents and tourists in the use of local resources and services (use of drinkable water and treatment of wastewater, air pollution, noise pollution, traffic, crowding, etc.)

Thence, to assure sustainable development of the tourism sector, it is important that the planning of tourism offered to a destination is based on a robust analysis of environmental, social and economic conditions of the area and of the current and potential impact of tourist activities, in comparison with the carrying capacity of the destination. According to this purpose, the assessment of the EF of tourists and of the biocapacity of the area represent an attempt to provide a supporting tool to decision makers, with the aim of addressing tourism 
strategies for the future development of the destination in a more sustainable way. Moreover, the aim of the present study is to analyze the impact of tourism on a destination, and to compare the sustainability of different kinds of holiday (due, for instance, to energy consumption or to the extension of built-up land), to evaluate the current situation, and to identify solutions that assure the development of tourism with fewer environmental costs.

\section{Methodology}

The ecological footprint (EF) method was developed in firstly in the 1990s by the ecologist William Rees from the British Columbia University, and then deepened and applied by Wackernagel and Rees [7], from the Ecological Footprint Network. EF is an aggregated index that correlates the life-style of a population with the amount of natural resources needed to support it (the "lifesupporting natural capital"). It is an indicator based on a basic concept, which is highly communicative because this relationship is measured by quite a simple parameter: the extent of the natural bioproductive area (measured in hectares per capita) needed to satisfy the consumption and to absorb the waste of a population. The assessment of the EF is based on an evaluation of consumption, clustered in five components: food, housing, transportation, goods and services. Any of these components can be responsible for an EF, which is measured in a specific unit of measure that is equal to one hectare of the mean productivity of the earth. The EF is the sum of six different kinds of land: Cropland, Pasture, Forest, Fisheries, Energy land, Built-up land. To determine if the EF of a community is sustainable or not, it is necessary to compare the local extension of bioproductive land (biocapacity) with the local demand for land (EF), defining an environmental balance of the local system. Assuming that to be sustainable a system has to be self-sustaining, the amount of deficit or overshoot of natural resources emerging from the environmental balance represents an estimation of the level of sustainability of the area considered.

\subsection{Ecological footprint of tourism}

Assessing the EF of tourism enables one to evaluate the overall impact of a holiday, involving not only the impacts due to the stay in a hospitality structure, but also, for instance, the impact due to the trip from home to the destination and back.

There are two main accounting methods for EF assessment (Monfreda et al. [5], Wackernagel et al. [8], Lewan et al. [3]):

- the compound model ("top-down" approach): the assessment is based on the sum of the EF of the consumption of an area (estimated using the aggregate national data about production and trade);

- the component model ("bottom-up" approach): the assessment is based on the sum of the EF of all relevant components of a population's resource consumption and waste production. 
The second is considered a more comprehensive and robust approach because the components of consumption of the community are considered in a whole and double counting is avoided. Nevertheless, it is not possible to apply this method for the assessment of the EF of a tourist destination because it is a peculiar system, for which it is not possible to obtain aggregate data. For this reason, in the present study the component model is applied: the consumption of every single person is estimated, considering the sum of all relevant components of consumption, and then the EF of the area is evaluated, multiplying this value for the overall number of people in the area (residents and tourists).

The phases of the evaluation are:

1. Assessment of the EF of residents.

2. Assessment of the EF of tourists.

3. Assessment of the biocapacity of the area.

4. Comparison between the sum of the EF of residents plus the EF of tourists and the local biocapacity, to evaluate the sustainability of the area.

Previous assessment of the EF according to the component model utilized the spreadsheet "Household Ecological Footprint Calculator", v. 3.2, 2003, developed by Redefining Progress (see, for example, Johnson [2]). In this study, the spreadsheet, developed for a Canadian assessment, was fitted to the Italian situation and to an assessment of potential impact of tourism at the local scale, to highlight how tourists' choice could affect the quality of the environment in a tourist destination. Thence, some standards about the local situation contained in the equations of the model were modified and new values from literature and statistical data on the Italian situation were introduced (e.g. mean size and age of houses, characteristics of roads and railways, etc.), to develop a spreadsheet useful to assess the EF in a local area in Italy. Furthermore, each item of the spreadsheet was associated with an item of official Italian statistical data, to assure the complete repeatability of the evaluation. Afterwards, starting from the result of the previous phase, the spreadsheet was further modified, to be adjusted to the consumption of a tourist during his holiday (trip, stay, food, excursions, etc.): some items that were not relevant for tourism activities were removed (e.g. goods and services not directly related with tourism) and then the model was modified to allow the evaluation of different kinds of holidays.

The research about methodology lead to the development of:

a) A model to assess the EF of Italian residents at the local scale

b) A model to assess the EF of tourists according to the kind of accommodation they choose for their holiday. This model is composed of the following sections:

- The EF of trip depending on the means of transport chosen (plane, car, motorbike, train, bus, bicycle)

- The EF of one night spent in a $1 *-2 *$ hotel

- The EF of one night spent in a $3^{*}$ hotel

- The EF of one night spent in a $4^{*}$ hotel

- The EF of one night spent in a camping site

- The EF of one night spent in an agritourism facility 
- The EF of one night spent in a B\&B

- The EF of one night spent in a second home (owned or rented)

- The total EF of the holiday (the EF of accommodation * no. of nights + the EF of the trip)

\subsection{Biocapacity}

To evaluate the result of EF the assessment of a specific area, it is necessary to compare this value, representing the human demand on nature for that area, with the amount of natural capital stock that the area can supply. In this perspective, local biocapacity represents the reference value to determine if human consumption is in a condition of deficit or overshoot in comparison with the natural resources availability.

The biocapacity of the area is evaluated assigning factors of bioproductivity (equivalence factors) to every type of land. The first step in the evaluation of biocapacity at the local scale is then the comparison between the classes of soil use available in local classification (in this case, the DUSAF database by Lombardy Region) with the six classes of land defined by the model developed by Redefining Progress. The result of this comparison is summarized in Table 1

Table 1: $\quad$ Comparison between land classes in the ecological footprint model and in DUSAF database.

\begin{tabular}{|c|c|c|c|}
\hline \multicolumn{2}{|c|}{$\begin{array}{l}\text { Land in Ecological } \\
\text { Footprint model }\end{array}$} & DUSAF classes of land use & $\begin{array}{l}\text { Equivalence } \\
\text { factor }\end{array}$ \\
\hline \multicolumn{2}{|c|}{ Energy land and forest } & Natural vegetation, woodland, woody plants & 1,34 \\
\hline \multicolumn{2}{|l|}{ Pasture } & Pasture and meadows & 0,49 \\
\hline \multirow[t]{2}{*}{ Cropland } & primary & $\begin{array}{l}\text { land under cultivation (arable crops, orchards, } \\
\text { vineyards, horticultural land) }\end{array}$ & 2,21 \\
\hline & marginal & Uncultivated land & 1,79 \\
\hline \multicolumn{2}{|c|}{ Built up land } & $\begin{array}{l}\text { serviced and infrastructured land, urban decay, } \\
\text { mining land }\end{array}$ & 2,21 \\
\hline \multicolumn{2}{|l|}{ Fisheries } & Lakes, basins, river beds e artificial watercourse & 0,36 \\
\hline & & $\begin{array}{l}\text { infertile soil, sandy lands, gravely soil, beaches, } \\
\text { detritical deposits, rocky outcrop without plant } \\
\text { cover }\end{array}$ & 0,00 \\
\hline
\end{tabular}

The last class in the table ("non productive land") is an integration in respect of the model developed by Redefining Progress: some classes of land use in the DUSAF database have no correspondence with land classification in the EF model and, moreover, they have a productivity equal to 0 . For this reason, this new class was created, with an equivalence factor equal to 0 . By assigning equivalence factors to the areas included in the respective DUSAF classes associated in the table, it is possible to estimate total biocapacity (expressed in global hectares or global square meters (Every kind of land has a different 
bioproductivity. To have the EF results expressed in a unique measure - the global hectare - the model normalizes the values of bioproductivity of the areas in different nations and of different kind of land.)) of the area considered. Comparing the EF of tourists and residents with the local biocapacity enables one to assess the impact of tourism in this area in relation to local resources, and to evaluate the sustainability of the destination.

Finally, the results of every single evaluation of the EF of tourism in every hospitality structure is summed up to assess the total EF of tourism in Alpi Lepontine and, then, this last result was added to the value of the EF of residents to be compared with the local biocapacity.

The EF of tourism was calculated with the following equation:

$$
\sum_{i}\left(E F_{i} * O_{i}\right)+\left(E F_{t} * T\right)
$$

$\mathrm{EF}_{\mathrm{i}}=$ the $\mathrm{EF}$ of an overnight spent in the $i$ type of hospitality structure

$\mathrm{O}_{\mathrm{i}}=$ the number of overnights per year spent in the $i$ kind of structure in the area considered

$\mathrm{EF}_{\mathrm{t}}=$ the mean $\mathrm{EF}$ of a trip to Alpi Lepontine (forward and back) per tourist $\mathrm{T}=$ the total number of tourists in the destination in the considered year

\section{Area of study}

The destination object of the study is Alpi Lepontine Mountain Community (Italian Mountain Communities are administrative clusters of municipalities in mountain areas), which is an area in the Lombardy Region in Northern Italy, near Switzerland. Alpi Lepontine represents an interesting situation because it has undertaken the process of European Charter for Sustainable Tourism in Protected Areas promoted by Europarc (European Federation of Parks), to promote sustainable tourism. In this context, the evaluation of the EF of tourism in the area and the assessment of the ecological deficit or overshoot of the whole area is an attempt to provide a valuable tool for policy makers to set targets for sustainable policies of development and to verify the ecological implications of policy choices.

There are also some specific characteristics of Alpi Lepontine that identify this destination as an interesting area for EF evaluation: first, the problem of soil exploitation and excessive urbanization, underlined as a crucial alarm by both residents and tourists (Castellani et al. [1], Tarelli et al. [6]), suggests the importance of an indicator that emphasizes the necessity of bioproductive land to support human consumption of goods and services; secondly, a questionnaire survey among tourists highlighted the importance of nature, and especially protected areas, as a key resource and a key factor of attraction for this destination: the presence of tourists that are more "environmentally friendly" represents a solid base for a communication campaign, based on the EF results, to address tourist choices (e.g. kind of accommodation, means of transport, etc.) towards more sustainable behaviour. 


\section{Results and discussion}

The main results of the implementation in Alpi Lepontine area are summarized in Table 2.

As is shown in Table 2, the EF of Alpi Lepontine largely exceeds the biocapacity of the area, highlighting a deficit between human demand on nature and natural stock: it means that the Alpi Lepontine area would not be able to support its level of consumption without depending on external resources. Although the EF of tourists is quite large, it is still smaller than the footprint of residents, therefore the action towards sustainability should involve both tourism and local activities. Comparing the daily footprint of residents and the daily footprint of tourists, it is possible to notice that even the smallest footprint of a tourist $\left(551 \mathrm{gm}^{2} /\right.$ day) is higher than the resident's one $\left(103 \mathrm{gm}^{2} /\right.$ day $)$ : it depends on the fact that the impact of hospitality structures derives from the number of beds the structure can offer but the number of tourists effectively present during a single day, especially in non-tourist season, is rarely equal to the maximum; on the other hand, the impact of a house or property has to be divided for the number of people living in that house, which is often proportional to the house size. In addition, hospitality structures remain on the territory for the whole year, but the presence of tourists is often condensed into only a short period of the year, so the territory has a benefit from them for only for a part of the year, but their footprint stays for the whole year.

Table 3 shows the results of the footprint assessment for a night spent in the different kinds of accommodation that can be chosen by Alpi Lepontine tourists:

Table 2: Main results of the EF and biocapacity assessment in Alpi Lepontine (gha/year).

\begin{tabular}{|c|c|c|c|}
\hline Biocapacity & EF of residents & EF of tourists & $\begin{array}{c}\text { EF residents + } \\
\text { EF tourists }\end{array}$ \\
\hline 3.693 & 78989 & 18684 & 97673 \\
\hline
\end{tabular}

Table 3: The EF of an overnight spent in different kinds of accommodation ( $\mathrm{gm}^{2} /$ day).

\begin{tabular}{|l|c|c|c|c|c|c|c|}
\cline { 2 - 9 } \multicolumn{1}{c|}{} & \multicolumn{7}{c|}{ HOSPITALITY STRUCTURE } \\
\hline $\begin{array}{l}\text { FOOTPRINT } \\
\text { [gm2] }\end{array}$ & H. $1 *-2 *$ & H. $3 *$ & H. $4 *$ & $\begin{array}{c}\text { Second } \\
\text { house }\end{array}$ & $\begin{array}{c}\text { Agritouris } \\
\mathrm{m}\end{array}$ & B\&B & $\begin{array}{c}\text { Camping } \\
\text { site }\end{array}$ \\
\hline FOOD & 0,36 & 0,36 & 0,36 & 0,36 & 0,36 & 0,36 & 0,36 \\
\hline HOUSING & 24,78 & 59,21 & 597,22 & 197,00 & 36,83 & 15,67 & 1248,89 \\
\hline $\begin{array}{l}\text { LOCAL } \\
\text { TRANSPORT }\end{array}$ & 9,98 & 9,98 & 9,98 & 9,98 & 9,98 & 9,98 & 9,98 \\
\hline GOODS & 1,56 & 1,56 & 1,56 & 1,56 & 1,56 & 1,56 & 1,47 \\
\hline SERVICES & 516,99 & 516,99 & 516,99 & 516,99 & 516,99 & 516,99 & 0,00 \\
\hline WASTE & 7,15 & 7,15 & 7,15 & 7,15 & 7,15 & 7,15 & 7,15 \\
\hline TOTAL EF & $\mathbf{5 6 0 , 8 2}$ & $\mathbf{5 9 5 , 2 5}$ & $\mathbf{1 1 3 3 , 2 5}$ & $\mathbf{7 3 3 , 0 4}$ & $\mathbf{5 7 2 , 8 7}$ & $\mathbf{5 5 1 , 7 0}$ & $\mathbf{1 2 6 7 , 8 5}$ \\
\hline
\end{tabular}


$1 *-2 *$ hotel, $3 *$ hotel, $4 *$ hotel, camping site, agritourism, B\&B and second houses. The accommodation that assures the smallest $\mathrm{EF}$ is $\mathrm{B} \& \mathrm{~B}$, while the biggest footprint comes from a night spent in a camping site (especially because of the footprint from housing, as explained further in the paragraph) and a $4^{*}$ hotel (especially because of energy consumption for services).

The analysis of the single components of the footprint highlights that housing is the most relevant component that discriminates between the footprint of different kinds of accommodation: once more, the camping site and $4 *$ hotel are the most expensive accommodation according to this parameter. In camping sites the footprint of housing is highly related to the soil use ("built-up" land) but it has to be considered that this land, although no more bioproductive (e.g. not available for agriculture or pasture), is not entirely built-up and can still provide some ecological functions (e.g. water runoff, carbon absorption or recreational use); regarding $4^{*}$ hotels, the main contribution to the footprint comes from energy consumption. Indeed, a focus on the single items of the footprint in the component "housing" highlights that energy consumption rises in hotels and non-hotel structures as the level of services increase (form B\&B to $4^{*}$ hotel); the only exception is the second house, because of the high amount of energy necessary to create building materials, and especially cement, for a structure which is often similar to the size of a $1 *-2 *$ hotel, but is used only by a few people and for a shorter period. To reduce the impact of this kind of hospitality structure, it would be useful to promote the use of these houses as guest accommodation.

Table 4: The EF on energy land by housing component in different structures.

\begin{tabular}{|c|c|}
\hline $\begin{array}{c}\text { HOSPITALITY } \\
\text { STRUCTURE }\end{array}$ & $\begin{array}{c}\text { HOUSING - ENERGY } \\
\text { [gm2] }\end{array}$ \\
\hline Bed \& Breakfast & 12,06 \\
\hline Agritourism & 13,53 \\
\hline Camping site & 14,91 \\
\hline Hotel 1 - 2 $*$ & 15,74 \\
\hline Hotel $3 *$ & 32,09 \\
\hline Hotel $4 *$ & 66,34 \\
\hline Second house & 186,72 \\
\hline
\end{tabular}

It is not possible to compare the footprint of food because, due to the nonavailability of specific data on food consumption (e.g. from specific questionnaires on the territory), the input values for food consumption are the same for the whole study (both for residents and tourists) and are obtained from national statistics on food consumption.

The footprint of goods is similar in all structures except for the camping site, because this item depends mainly on the footprint of furniture in the bedroom, which is quite similar in all kinds of hotel bedrooms (especially because Italian law gives some standard rules about the furniture in hotel rooms) and it is not present in a camping site. 
As for goods, the footprint of services is also quite similar in the hotel and non-hotel structures, except for the camping site. This difference is due to the fact that the model developed includes three kinds of services in the footprint calculation (wastewater disposal, laundry and phone services) but data were available only for one of them, the laundry service, which is often self-organized by tourists in the camping site, and can't be considered a structured service, used by all the tourists. It is important to notice that in hotel structures (and often in agritourism) the laundry service could concern also the restaurant (not only the linen for the bedrooms), but in many cases the restaurant is not only dedicated to the resident tourist, so it would be difficult to identify the footprint of this service for each resident tourist; for this reason, the footprint of the laundry service is calculated only for the linen of the bedrooms.

As for food consumption, detailed data about waste production for different kinds of accommodation are not available, so in this study the amount of waste for every single night spent in a hospitality structure was considered equal to the amount of waste produced by a local resident during one day. It is of course a significant approximation, because every kind of accommodation has different services and different management, so for future studies it would be very interesting to deepen the analysis on this topic, trying to define a specific relation between the type of accommodation chosen by tourists and the amount of waste generated per tourist.

The EF of local mobility, due for instance to daily excursions, is evaluated on the basis of a local survey about tourists' behaviour, and is therefore the same for every type of accommodation.

The assessment of the EF of the component "transportation", which consists of the trip from home to the destination and back (local transfer by tourists is included in the daily footprint), was developed separately from the assessment about accommodation, because these two kinds of choices are independent. Considering that the EF of a trip depends on the distance of the starting point from Alpi Lepontine, which is different for every tourist, a mean value per tourist was calculated, based on a weighted distance estimated from statistic tourist data (number of tourists from every region and country) and on a percentage distribution of mean of transport use (investigated by specific questionnaires and analysis).

\section{Conclusions}

Ecological footprint methodology application could be useful to understanding the impact of several kinds of human pressure and their capability to be "sustained" by a territory. The assessment of tourism impacts done through the evaluation of the EF of a tourist destination involves three categories of subjects: tourists, tourism operators and local administrators. Each kind of subject indeed can act for the development of tourism in a more sustainable perspective:

- tourists can choose different aspects of their holiday: kind of accommodation, length of stay, means of transport, local or international food, etc; 
- $\quad$ tourism operators can act to make tourism structures more ecologically efficient, reducing impacts due to energy and water consumption, production of waste, etc.;

- $\quad$ public administrators are involved in the definition of land use planning and can influence the whole tourism system, promoting communication campaigns to raise environmental consciousness among residents, tourism operators and tourists, and supporting the development of the destination from a sustainable perspective.

A further implementation of the methodology requires a better evaluation of waste production and energy consumption (included in the model but still lacking more detailed information) and the evaluation of water consumption (not yet included in the original model).

\section{Acknowledgements}

This research was undertaken within the project "Sovvenzione Globale INGENIO", funded by the European Social Fund, the Italian Ministry of Labour and Social Policy and by the Lombardy Region.

\section{References}

[1] Castellani, V., Lombardo, A., Sala, S. Strategy for sustainable mountain tourism. A private - public synergy for local development. In: Proceedings of III International Seminar on Mountain Tourism "Sustainable Tourism in mountain protected areas - building partnership between NGOs and managers of protected areas", Sucha Beskidzka, Poland, 25- 28 October 2007 , in press

[2] Johnson, P. A., Exploring the Ecological footprint of tourism in Ontario, University of Waterloo, Canada. 2003

[3] Lewan, L., Simmons, C. The use of Ecological footprint and biocapacity analyses as sustainability indicators for sub-national geographical area: a recommended way forward, Ambiente Italia, Italy. 2001

[4] May, V., Tourism, the environment, and development: values, sustainability and stewardship. Tourism Management, 12(2), pp. 112-118, 1991

[5] Monfreda, C., Wackernagel, M., Deumling, D. Establishing national natural capital accounts based on detailed Ecological footprint and biological capacity accounts. Land Use Policy, 21 (2004) 231-246, 2004.

[6] Tarelli, E., Castellani, V., Leoni, L., Sala, S. Via Regina: discovering the past for a sustainable future. Strategy and Action Plan 2008-2012. Technical report for the implementation of European Charter for Sustainable Tourism in Protected Areas. 2008

[7] Wackernagel M., Rees W. E. Our Ecological Footprint: reducing human impact on the Earth, New Society Publishers, Canada. 1998

[8] Wackernagel M., Monfreda, C., Moran D., Wermer, P., Goldfinger, S., Deumling, D., Murray, M. National Footprint and Biocapacity Accounts 2005: the underlying calculation method, Global Footprint Network, Oakcland, USA. 2005 